\title{
Review: albumin administration is not associated with excess mortality in acutely ill patients
}

Wilkes MM, Navickis RJ. Patient survival after human albumin administration. A meta-analysis of randomized, controlled

trials. Ann Intern Med 2001 Aug 7;135:149-64.

\section{QUESTION: Is albumin administration associated with excess mortality in acutely ill patients?}

\section{Data sources}

Published and unpublished trials were identified by searching Medline, EMBASE/Excerpta Medica, the Cochrane Controlled Trials Register, and the Cochrane Medical Editors Trial Amnesty of unpublished trials; by searching Altavista, Northern Light, HotBot, and Excite search engines for relevant internet resources; by handsearching JAMA, New England Journal of Medicine, Lancet, and BMJ from January 1990 to November 2000; contacting albumin suppliers and authors of published randomised trials; and reviewing bibliographies of previous meta-analyses, review articles, and other investigations involving albumin. Studies in any language were considered.

\section{Study selection}

Studies were selected if they were randomised controlled trials comparing intravenous albumin treatment with crystalloid treatment, no purified albumin, or a lower dose of purified albumin; and had available mortality data. There were no restrictions on the clinical indication for albumin administration or therapeutic intent. Studies were not excluded if concomitant treatments were given in a similar manner to both study groups. Studies were excluded if the control group tested synthetic colloids, blood products, or plasma protein fraction.

\section{Data extraction}

Data were extracted on clinical setting, study population, details of albumin and control regimens, study endpoints, study quality, and outcomes. Main outcome measure was mortality.

\section{Main results}

55 trials ( $\mathrm{n}=3504)$ met the inclusion criteria. Duration of follow up was available for 49 trials; median duration of follow up was 11 days (range 0.04 to $1096 \mathrm{~d}$ ). 13 trials were not included in the pooled analysis because no patients died in either group. Of the remaining 42 trials, there was no statistically significant difference in mortality between intervention and control groups (table). The relative risk of death was lower in trials that had blinding, $\geqslant 100$ patients, mortality as an endpoint, and in those without crossovers.

\section{Conclusion}

In acutely ill patients, albumin administration is not associated with differences in mortality.

Sources of funding:

Plasma Protein

Therapeutics Association

and American Red

Cross.

For correspondence Dr M M Wilkes, Hygeia Associates, 17988 Brewer Road, Grass Valley, $C A$ 95949, USA. Fax +1 5302682301 .

A modified version of abstract and commentary appears in ACP Journal Club.

Albumin v control for mortality in acutely ill patients*

\begin{tabular}{llll} 
& \multicolumn{2}{c}{ Weighted event rates } & \\
\cline { 2 - 3 } Trial categories & Albumin $v$ control & RR (95\% Cl) & RRI (CI) \\
\cline { 1 - 3 } All & $19 \% v 17 \%$ & $1.11(0.95$ to 1.28$)$ & $11 \%(-5$ to 28$)$ \\
\hline Surgery or trauma & $12 \% v 11 \%$ & $1.12(0.85$ to 1.46$)$ & $12 \%(-15$ to 46$)$ \\
\hline Burns & $27 \% v 15 \%$ & $1.76(0.97$ to 3.17$)$ & $76 \%(-3$ to 217$)$ \\
\hline Hypoalbuminaemia & $15 \% v 10 \%$ & $1.59(0.91$ to 2.78$)$ & $59 \%(-9$ to 178$)$ \\
\hline High risk neonates & $24 \% v 20 \%$ & $1.19(0.78$ to 1.81$)$ & $19 \%(-22$ to 81$)$ \\
\hline & & & RRR (Cl) \\
\hline Ascites & $27 \% v 29 \%$ & $0.93(0.67$ to 1.28$)$ & $7 \%(-28$ to 33$)$ \\
\hline Other & $26 \% v 29 \%$ & $0.91(0.67$ to 1.22$)$ & $9 \%(-22$ to 33$)$ \\
\hline
\end{tabular}

*Abbreviations defined in glossary; RRI, RRR, and $\mathrm{Cl}$ calculated from data in article. All meta-analyses were done using a fixed effects model.

\section{COMMENTARY}

The review by Wilkes and Navickis is the $13^{\text {th }}$ meta-analysis evaluating the effect of fluids on mortality in seriously ill patients. The strengths of this meta-analysis are the comprehensive search strategies used to identify primary studies, which minimised publication and English language bias; the explicit selection criteria used; the use of methodological quality, such as random assignment, as an inclusion criterion; extraction of data in duplicate; and rating of treatment allocation, crossovers, and blinding for each included study. However, all relevant co-interventions, such as blood products, were not well reported in the original trials. 23 of 78 potentially eligible studies were excluded because no mortality data were available, highlighting how many trials were designed to address short term physiological endpoints.

In this meta-analysis, the relative risk (RR) of mortality for all patients was 1.11 (table). Subgroup analyses were planned $a$ priori, and included surgery or trauma patients, those with burns or hypoalbuminaemia, and neonates (RRs shown in table).

This review is relevant to all clinicians caring for seriously ill patients. Results for the overall population and for all but one of the subgroups indicate a trend toward harm associated with albumin; the relative risks are $>1$. The most sanguine result is seen for patients with ascites, although the confidence interval includes the possibilities of modest benefit and modest harm, as also shown for the other subgroups (table). In deciding on the use of albumin in critically ill patients, clinicians should consider the lack of evidence of benefit, and the trend toward harm in the point estimate, and the confidence interval including the possibility of an appreciable mortality increase, shown in this and other meta-analyses. These factors, and the cost of albumin compared with crystalloids, ${ }^{1}$ may have contributed to decreased use of albumin in some sectors. ${ }^{2}$ Meanwhile, the international research community has embarked on additional focused physiological studies and modern, large rigorous randomised trials in diverse populations to more precisely understand the effect of albumin on morbidity and mortality.

Deborah Cook, MD, MSc, Professor Gordon Guyatt, MD, MSc, Professor Medicine and Clinical Epidemiology and Biostatistics McMaster University, Hamilton, Ontario, Canada

1 Cook D, Guyatt G. Colloid use for fluid resuscitation: evidence and spin [editorial]. Ann Intern Med 2001;135:205-8.

2 Roberts I, Edwards P, McLelland B. More on albumin. Use of human albumin in UK fell substantially when systematic review was published [letter]. BMJ 1999;318:1214-5. 